\title{
Physiological Adjustments to Stress Measures Following Massage Therapy: A Review of the Literature
}

\author{
Albert Moraska ${ }^{1,2}$, Robin A. Pollini ${ }^{1,3}$, Karen Boulanger ${ }^{1,4}$, Marissa Z. Brooks ${ }^{1,5}$ \\ and Lesley Teitlebaum ${ }^{1}$
}

${ }^{1}$ Massage Therapy Foundation, Evanston, Illinois, ${ }^{2}$ University of Colorado at Denver and Health Sciences Center, School of Nursing, Denver, Colorado, ${ }^{3}$ University of California at San Diego, School of Medicine, San Diego, California, ${ }^{4}$ University of lowa, Community and Behavioral Health, lowa City, lowa and ${ }^{5}$ Portland State University, Portland, Oregon, USA

Use of massage therapy by the general public has increased substantially in recent years. In light of the popularity of massage therapy for stress reduction, a comprehensive review of the peer-reviewed literature is important to summarize the effectiveness of this modality on stressreactive physiological measures. On-line databases were searched for articles relevant to both massage therapy and stress. Articles were included in this review if (i) the massage therapy account consisted of manipulation of soft tissues and was conducted by a trained therapist, and (ii) a dependent measure to evaluate physiological stress was reported. Hormonal and physical parameters are reviewed. A total of 25 studies met all inclusion criteria. A majority of studies employed a 20-30 min massage administered twice-weekly over 5 weeks with evaluations conducted pre-post an individual session (single treatment) or following a series of sessions (multiple treatments). Single treatment reductions in salivary cortisol and heart rate were consistently noted. A sustained reduction for these measures was not supported in the literature, although the single-treatment effect was repeatable within a study. To date, the research data is insufficient to make definitive statements regarding the multiple treatment effect of massage therapy on urinary cortisol or catecholamines, but some evidence for a positive effect on diastolic blood pressure has been documented. While significant improvement has been demonstrated following massage therapy, the general research body on this topic lacks the necessary scientific rigor to provide a definitive understanding of the effect massage therapy has on many physiological variables associated with stress.

Keywords: catecholamines - complementary medicine - cortisol - manipulative medicine

\section{Introduction}

Stress is the disruption in homeostasis caused by psychological experiences or physiological perturbations (1). Even so-called healthy living involves continual exposure to a variety of stressful experiences; however, the body addresses these in an allostatic manner

For reprints and all correspondence: Albert Moraska, University of Colorado at Denver and Health Sciences Center, School of Nursing, C288, Education 2 North 13120 east $19^{\text {th }}$ Ave. Aurora, CO 80045.

E-mail: moraska@alum.rpi.edu thereby maintaining homeostasis. Physiological changes in response to a stressor such as epinephrine or cortisol production and release, prepare the individual to better cope with the situation. Minutes or hours following a stressful event the system usually returns to homeostasis. However, when the demands on the individual to manage the stressful experience exceed the resources available, acute stress may precipitate negative psychological and/or physical symptoms. Both severe stressors (e.g. major life events) and relatively minor stressors that occur on a frequent basis (e.g. daily hassles) have 
been associated with an increase in stress-related symptoms $(2-5)$.

When stressful experiences become chronic, pathological consequences result and can contribute to physiological or psychological dysfunction including high blood pressure, cardiovascular disease, reduced immune function, thyroid disorders, alcoholism, diabetes, anorexia nervosa as well as depression and increased suicide attempts $(6,7)$. In fact, in the United States the five leading causes of death have been linked to stress: heart disease, cancer, lung ailments, cirrhosis of the liver and suicide (8). Therefore, therapeutic techniques that reduce the harmful consequences of stress and facilitate effective coping mechanisms are essential to healthy living.

Complementary and alternative medicine (CAM) approaches to address health issues has become increasingly popular in recent years $(9,10)$. It has been estimated that $36-42 \%$ of the US population use CAM modalities, with $5-11 \%$ specifically seeking massage therapy $(9,11)$. Eisenberg et al. (9) estimated that 114 million visits to US-based massage therapists were made in 1997 exceeding those to all other CAM providers, with the exception of chiropractors. While reasons for seeking massage therapy are diverse, visits are frequently related to stress reduction. For example, wellness care (relaxation) accounted for $19 \%$ and anxiety reduction $5-9 \%$ of total visits to massage therapists (12).

The stress response is the body's reaction to any real or perceived threat and activates the sympathetic branch of the autonomic nervous system resulting in stimulation of the hypothalamus-pituitary-adrenal (HPA) axis $(13,14)$. Subsequent to HPA activation is the production of a cascade of stress-associated hormones including epinephrine (adrenaline) and cortisol, which influence other physiological systems and enhance an individual's ability to address the stressor. This mechanism of action will increase heart rate and mobilize fuel stores in preparation for the perceived threat; however, under chronic stressful conditions the negative consequences described previously ensue. In contrast, massage therapy is believed to stimulate the parasympathetic branch of the autonomic nervous system. Activation of the parasympathetic nervous system facilitates the return to homeostasis after an emergency by reversing some of the physiological systems activated during the stress response. For example, an increase in parasympathetic tone would reduce heart rate and slow respiration, resulting in relaxation.

Massage therapy has been touted as an antidote for the symptoms of stress by the research community (15), massage professionals (16) and the lay public (17). In an effort to more thoroughly understand if massage therapy is effective in the management of the negative health consequences of stress, a synthesis of the available scientific literature is needed. The intent of this review is to provide a comprehensive, yet critical, evaluation of the peer-reviewed research investigating the relationship between massage therapy and physiological measures of stress. Physiological measures of stress including hormonal (cortisol, epinephrine and norepinephrine) as well as physical (heart rate and blood pressure) are reviewed; study limitations and areas for future research are also discussed.

\section{Methods}

\section{Massage Definition and Inclusion Criteria}

We define massage therapy to be the manipulation of soft tissues for the purpose of producing physiological effects on the vascular, muscular or nervous systems of the body. It has been reported that the effect of massage therapy is more robust when administered by trained therapists (18), therefore, only studies employing trained massage therapists are included in this review. Studies in which partners, parents or a relative were the primary source for massage are excluded. In addition, inclusion of a research study requires direct tissue manipulation, as such, studies including only touch therapies (e.g. gentle touch, therapeutic touch, acupressure and reflexology) are excluded. Given the limitations in application of massage therapy to infants and children, we chose to exclude studies involving these populations. Because massage therapy treatment of short duration, applied only to a localized body region, of short duration is believed to be effective, we chose to include those studies provided they met all other selection criteria.

\section{Electronic Searches}

Searches for research manuscripts included the following: MEDLINE, CINAHL, PsychINFO and the Massage Therapy Foundation database. In addition, the authors' personal libraries and relevant citations from selected articles were reviewed. Keywords used for electronic searches included 'stress' in combination with each of the following terms: 'massage', 'bodywork', 'physiotherapy' and 'manual therapy'.

\section{Article Inclusion}

Using the above search terms, a total of 1032 citations that were published by the end of 2006 were initially identified. Titles and abstracts from all 1032 citations were then reviewed for topical relevance; this process resulted in 122 unique citations that were more closely scrutinized. Review articles, Letters to the Editor, study summaries and non-research reports were excluded from further review. A total of 62 articles were subsequently read in their entirety with those articles selected for inclusion presenting dependent variables of stress that included the hormones cortisol, epinephrine, norepinephrine or physical measures of blood pressure (BP) 
and heart rate. Following that analysis, 25 articles were found to meet all inclusion criteria and were included in this report.

The intent of this synthesis was to assess the impact of massage therapy on physiological measures of stress, yet the published research had great variability in quality of study design and conduct. We therefore chose to review the literature that met our inclusion criteria described previously, rather than selecting articles based solely on strength of study criteria $(19,20)$. Only those studies published in peer-reviewed journals and written in English language were reviewed.

\section{Results}

\section{Summary of Included Studies}

A total of 25 studies met our inclusion criteria. Of those, 18 employed a randomized control trial (RCT) study design, two were conducted using a quasi-experimental design and five used a within-subjects design. Descriptions of the massage therapy interventions used were quite varied and ranged from a simplistic description of the session to a detailed presentation of techniques that could readily reproduce the treatment. The majority of the studies (22) administered massage therapy to subjects on a massage table, whereas three used chair massage. Duration of a massage session varied from 5 to $90 \mathrm{~min}$, with over half $(52 \%)$ of the studies using sessions lasting between 20 and $30 \mathrm{~min}$. In many studies, the intervention consisted of 6-10 massage treatments, but research studies reported data following a single massage to as many as 22 sessions. Data collection following the first massage treatment session was the time point most frequently assessed. Given the breadth in descriptiveness of the techniques coupled with the subsequent variability of study results we chose to not provide comments on specific massage techniques. Study populations were varied and included sexually abused women (21), patients with eating disorders $(22,23)$, pain conditions (24-26), hypertension (27,28), HIV positive diagnosis (29), cancer (30-32), post-operative patients $(31,33)$, critical care patients $(34)$, healthy adult populations (35-42) as well as specific disease states (43-45).

\section{Hormones}

\section{Cortisol}

The hormone cortisol has become synonymous with 'stress hormone'. Although circadian rhythm causes daily fluctuation for this variable, chronic stress will perpetually increase hypothalamic-pituitary-adrenocortical activity causing cortisol production to become elevated beyond normal levels. Multiple sampling sources have been evaluated for cortisol including saliva, urine and blood plasma. Studies that investigated the effects of massage therapy on these physiological measures are summarized in Table 1.

\section{Salivary Cortisol}

Saliva provides an easy, non-invasive means for assessing cortisol and is frequently reported in massage studies. While serum-free cortisol measures are the most reliable assessments for cortisol, salivary cortisol is accepted as an accurate window for this measure (46). Determination of a single-treatment effect of massage therapy is a commonly used experimental design in massage studies assessing salivary cortisol. Salivary cortisol evaluations are typically conducted at the first and final (typically 6th to 10th) massage sessions.

Of the nine studies reporting salivary cortisol at the first massage session, eight $(89 \%)$ note a significant reduction immediately following massage therapy (22$25,27,29,40,41)$. It is unclear how long this reduction persists since a time-course has not been reported. Yet, in all of the studies when assessment of salivary cortisol is taken later in the study, this measure has returned to the baseline value. Thus, reductions in salivary cortisol may be short lived and multiple massage treatments do not appear to have a cumulative effect, although specific investigation into this has not been conducted. In the eight studies where salivary cortisol is assessed immediately pre-post the final massage improvement is less frequent with $63 \%$ of the studies now reporting a significant reduction $(21,24,25,27,41)$. It is interesting that the one study that failed to find a reduction at the initial massage session reported a reduction at the final (eighth) session (21). The subject populations for studies reporting salivary cortisol are highly varied, which suggests that many groups may experience an immediate benefit from massage therapy for this variable; most study participants were, however, either healthy adults or experiencing chronic life stress. An example of the study design and findings for salivary cortisol is demonstrated in a study conducted by Lawler and Cameron (25) on migraine patients. In this study the authors assess cortisol before and after a $45 \mathrm{~min}$ massage following the first and sixth (final) massage sessions. Although the authors did not report cortisol data for the control group, they note a reduction in salivary cortisol from 6.25 to $3.86 \mathrm{nM}$ following the first massage. At the sixth session they report similar pre- and post-massage values.

\section{Urinary Cortisol}

While saliva has been used to assess a single treatment change in cortisol following massage therapy, urine has been used more frequently to assess changes following multiple massage treatments. In massage studies an assessment is typically made at baseline and after 5 weeks of twice-weekly massage. Thus, this measure 


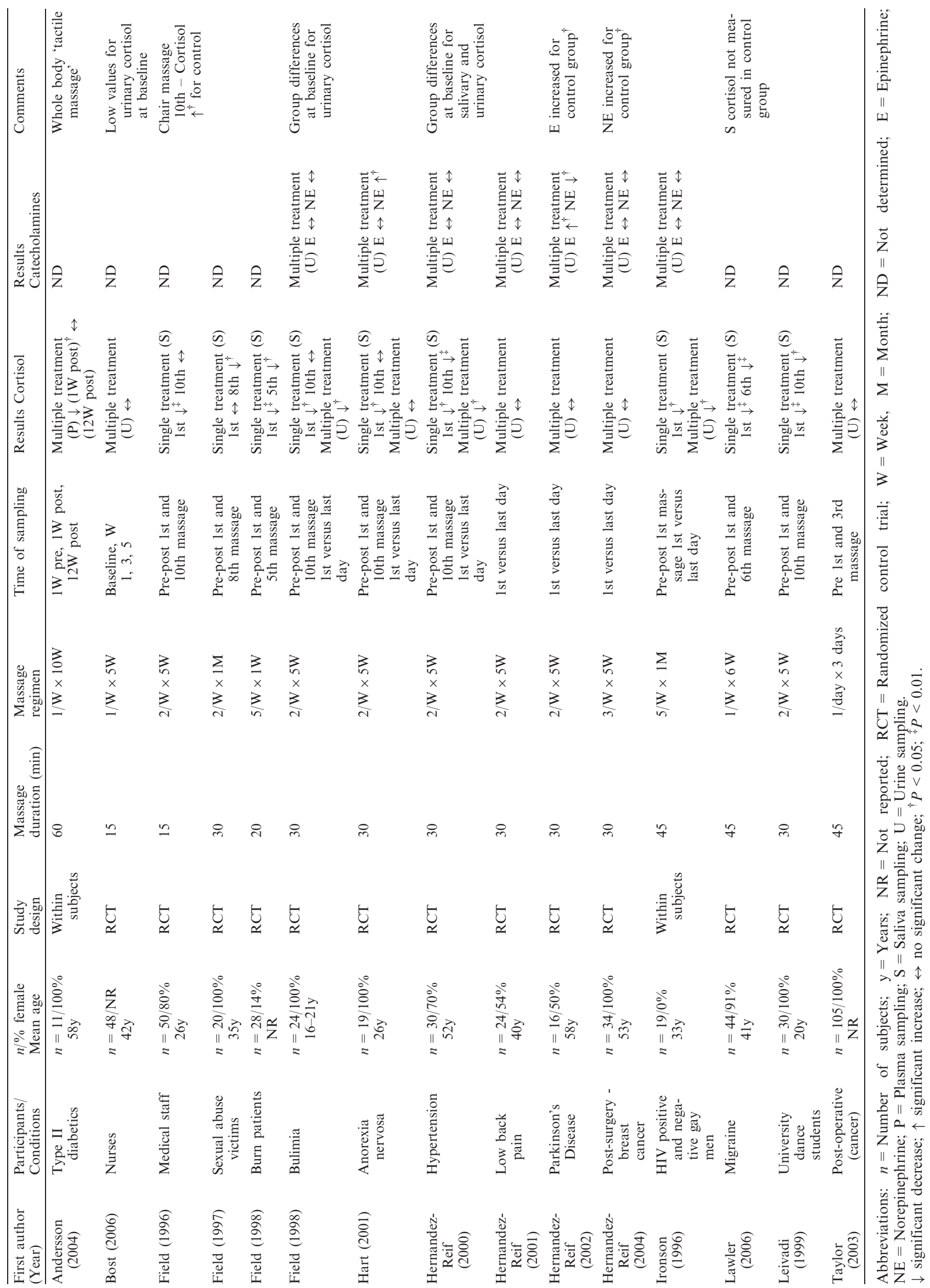


reflects the effect of multiple massage sessions over several weeks. With this method of assessment, three of nine published studies report a significant reduction in urinary cortisol following massage treatment $(22,27,29)$. However, baseline differences between massage and control populations weaken the conclusion for two studies $(22,27)$. In the third study where an effect was noted, massage therapy was administered on a daily basis for 22 consecutive days (29); in contrast, massage therapy given on three consecutive days was insufficient to establish an effect (33). The remaining studies all report no change in urinary cortisol $(23,26,31,33,36,43)$.

\section{Plasma Cortisol}

No comprehensive studies were located that assessed plasma cortisol in subjects receiving massage therapy. One uncontrolled pilot study applied a 'tactile massage', in which the skeletal muscles were not manipulated to 11 subjects and reported reduced plasma cortisol compared to baseline after 10 weekly massage sessions in subjects with Type II diabetes (44).

\section{Catecholamine}

Psychological stress can increase sympathomedullar and sympathoneuronal nervous system activity (47). Epinephrine (adrenaline) is produced mainly from the adrenal medulla and reflects the subject's sympathomedullar activity. Norepinephrine (noradrenaline) is considered an indicator of sympathoneuronal activity as most of the circulating norepinephrine is released from sympathetic nerve endings. This hormonal defense reaction is aimed at mobilizing energy for the muscles and heart while reducing blood flow to the internal organs and the gastro-intestinal system. Whereas epinephrine output is mainly influenced by mental stress, norepinephrine is more responsive to physical activity (47). A decrease in either of these variables may indicate a physiological reduction in stress. Studies that explore the relationship between massage therapy and catecholamines are discussed subsequently and summarized in Table 1.

\section{Epinephrine and Norepinephrine}

Seven studies report data on catecholamines following massage therapy, all of which determine levels of epinephrine and norepinephrine in urine samples at a similar periodicity as described for urinary cortisol $(22,23,26,27,29,31,43)$. In six of the studies reporting epinephrine no change was noted, while one study reported an increase in urinary epinephrine in patients with Parkinson's Disease (43). Norepinephrine was also largely unaffected by massage therapy as no change in this variable was reported in five studies $(22,26,27,29,31)$, while it increased in one report (23) and decreased in the remaining study (43). While the lack of an observed effect of massage therapy on catecholamines is striking, this may be due to the limited variability in study design. For instance, all of the studies originate from one research group and six use the same duration and frequency of massage treatment. In addition, only epinephrine and norepinephrine sampled from urine have been reported in the literature; no studies have reported the effect of massage therapy on plasma catecholamines.

\section{Blood Pressure and Heart Rate}

Increases in blood pressure, respiration and heart rate are all physiological manifestations of the sympathetic nervous system's response to stressful events. Stressful experiences in the workplace, for example, can raise BP and heart rate, which could subsequently contribute to cardiovascular disease. One argument is that massage therapy may ameliorate these symptoms of stress by promoting parasympathetic activity. Sixteen massage studies report data on $\mathrm{BP}$ or heart rate variables and are summarized in Table 2.

\section{Blood Pressure}

We identified eight studies that reported single-treatment effects of massage therapy on BP. Of these, three documented statistically significant reductions $(35,37,42)$, four indicated no change $(27,30,32,38)$ and one reported a reduction in mean arterial BP during, but not immediately after, massage (34). The collective body of literature reported participant systolic BP at baseline to be normal or mildly elevated, with the range between 117 and $144 \mathrm{mmHg}$. For the studies reporting statistical improvement in BP following a single massage session the change in systolic BP was relatively small $(2-12 \mathrm{mmHg})$. In one study that strictly focused on BP, the authors noted that a single 30 -min massage reduced systolic and diastolic BP by $4-8 \%$ (35). These effects were observed when massage therapy was directed to the back, neck and chest, whereas only systolic BP was reduced when massage therapy was to the legs, arms and face, suggesting that body region, rather than surface area may be an important consideration. In the three studies where diastolic BP was reduced, systolic BP also was reduced $(35,37,42)$. While $50 \%$ of the studies reported no effect after a single massage session, this includes a broad group of studies in which great variability in subject health and massage duration complicates accurate analysis. For example, a single 90 -min massage did not affect BP for healthy adults (38), however study participants entered massage treatment with normal systolic BP, thus the lack of an effect may be a desired finding.

Seven studies reported on changes in BP following multiple massage treatments or at a long-term follow-up after the final massage; their findings suggest a 


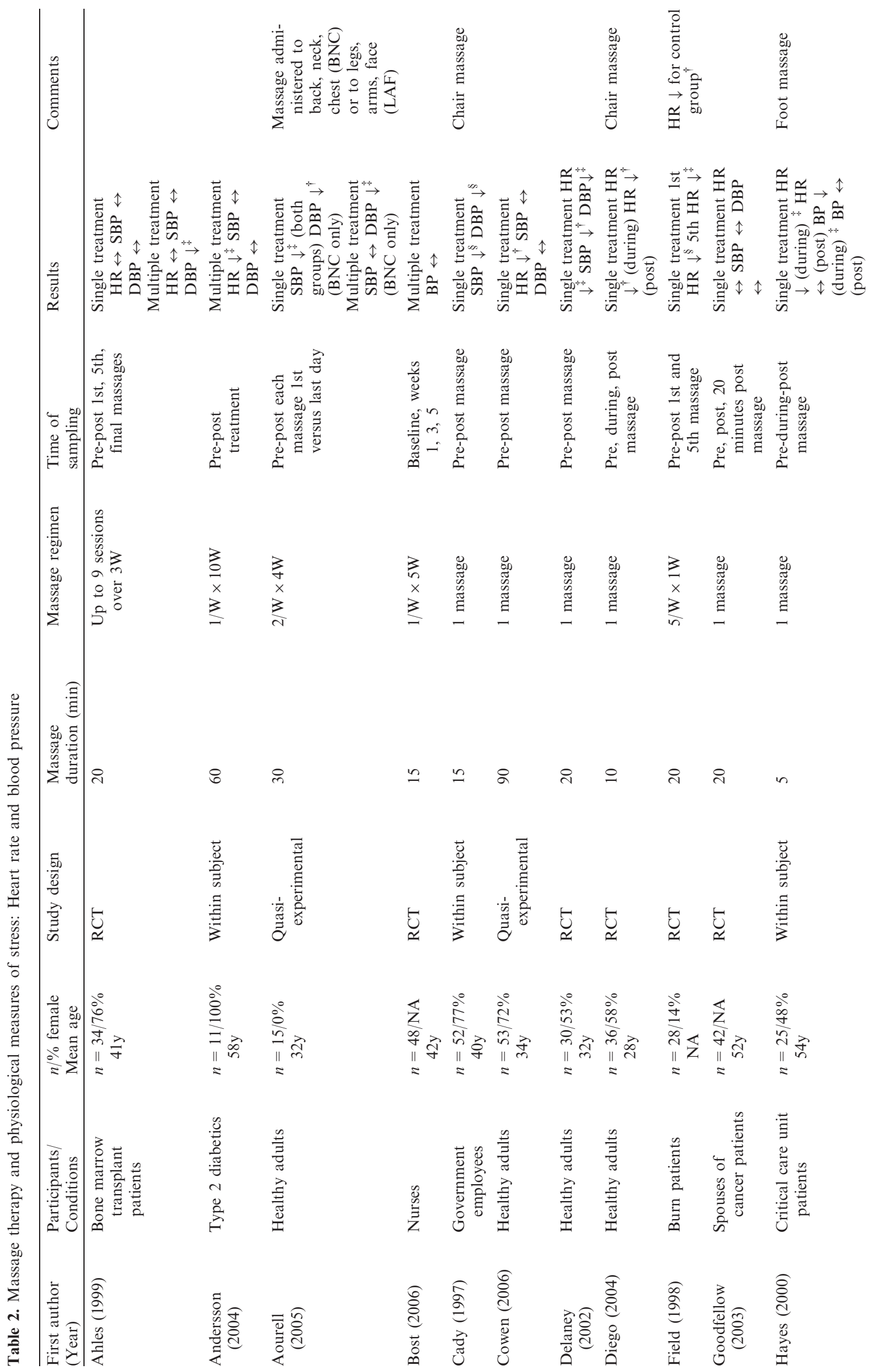




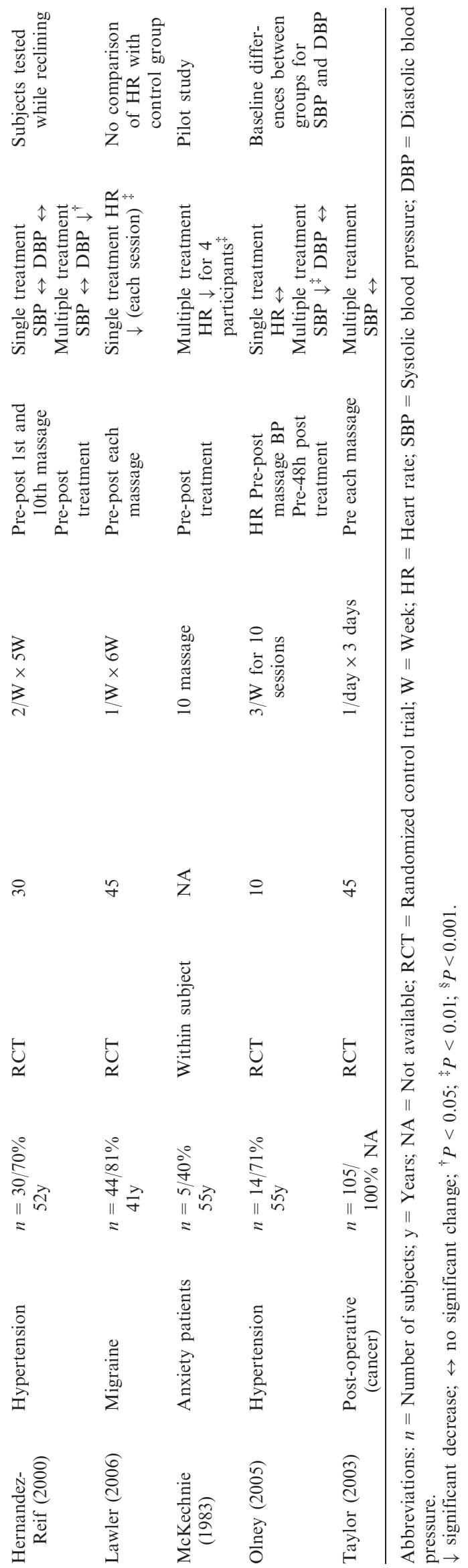

multiple-treatment effect of massage therapy on diastolic, but not systolic BP. In studies where eight or more massage sessions were administered over 3 or more weeks, subjects' diastolic BP was reduced in four studies $(27,30,35)$; this includes a study involving hypertensive participants. In the study of hypertensive patients, following 10 massage sessions a significant reduction in diastolic BP was noted when the subject was reclining, although they did not detect a reduction in BP following a single massage session unless the subject was in a sitting position during measurement (27). However, another study involving participants with hypertension reported a significant reduction in only systolic BP after 10 massage sessions (28). In general, the effect of multiple massage treatments on systolic BP appears to be less supported, while six studies report on this variable, only the one study noted previously reported a reduction in this measure (28). One small (11 subjects) within-subjects study did not find a change in systolic or diastolic BP (44) and a final study did not note a change in mean arterial BP (36). No studies reported an increase in BP following massage therapy.

\section{Heart Rate}

Heart rate (HR) was reported in 11 studies on massage therapy with nine reporting data following a single treatment and three following multiple treatments. An effect of a single massage treatment was reported in five studies $(24,25,38,39,42)$ with four reporting no change $(28,30,32,34)$. The studies identifying significant significant change note small, but statistically significant reductions in HR of approximately 3-6 beats per minute (bpm) immediately following massage therapy. This effect does not appear to persist beyond the massage session. For example, a 5-min foot massage administered to critical care patients effected a statistically significant decrease in HR from 97.3 to $94.7 \mathrm{bpm}$ during the massage, however, the reduction was not maintained when assessed $5 \mathrm{~min}$ post-massage (34). A longer massage treatment yields only slightly greater reductions in HR. Cowen et al. (38) found that a single 90-min Thai or Swedish massage significantly reduced HR among healthy subjects when assessed upon massage therapy completion (69.0 bpm to $63.4 \mathrm{bpm})$. Yet, the benefit may be repeatable as heart rate was reported to be reduced at each of six massage sessions for migraine sufferers, although the effect did not carry over to following sessions (25). In addition, a massage session that focused on myofascial trigger point therapy significantly reduced HR immediately after massage therapy (42). The authors also reported evidence indicating an increase in heart rate variability, which suggests the mechanism for reducing heart rate is by increasing parasympathetic activity. Although five studies (56\%) reported a decrease in HR following a single massage, it is important to note that 
one did not have a comparison control group (25), which prevents clarity regarding whether the effect was the result of massage therapy or, more simply, resting quietly. Uncertainty exists for another study where the authors document a significant reduction in HR for burn patients, but also note a significant reduction for a 'sit and relax' control group (24). While individual effects on HR may vary, collectively, a small decrease in resting HR is frequently observed immediately following massage therapy and the duration of the massage does not appear to be a critical variable. Yet, the effect does not persist as HR measurements taken just minutes following massage therapy have been reported to return to pre-massage values.

Three studies assessed HR following multiple treatments $(30,44,45)$; the two that report reductions in this variable were both small within-subjects studies $(44,45)$ with a total of 15 subjects in the two studies.

\section{Discussion and Conclusions}

This report reviewed the literature on the effects of massage therapy at reducing physiological measures of stress, including hormonal (cortisol, epinephrine and norepinephrine) as well as physical (heart rate and blood pressure) variables. Published research in this field has evaluated massage therapy following single or multiple treatments. The single treatment measurement evaluated a dependent measure immediately prior to and following a massage session, whereas multiple-treatment effects were determined prior to the first massage and following a series of sessions that occur over successive days or weeks. Our review of the literature indicated that massage therapy may have a beneficial effect on several physiological variables, specifically salivary cortisol and heart rate, when assessed immediately pre-post massage, but is null or inconclusive for the multiple-treatment effect on physiological indices of stress.

A reduction in salivary cortisol was evident following a single massage treatment, yet salivary cortisol returns to initial values when assessed at a later time point, even if massage therapy was administered during the interim timeframe. The single-treatment effect, however, recurred as successive massage sessions also showed to be generally effective at lowering salivary cortisol. Reduction in HR was also documented immediately after massage therapy and while statistically significant, only modest reductions were reported. This effect on HR did not appear to persist for long after the massage session, but the reductions were again repeatable. It should be noted that a sizable percentage $(44 \%)$ of the studies reporting HR did not detect a change in this variable. While several studies noted a reduction in BP from a single massage treatment, a greater number did not identify a significant change, although multiple-treatment effects on diastolic BP appeared to be more promising.

Hormonal variables associated with stress were largely unaffected by multiple massage treatments. Specifically, while as many as 10 massage sessions may have been administered between assessment time points, urinary measures of cortisol, epinephrine and norepinephrine were generally not affected by this treatment. However, the lack of an observed effect of massage therapy on these variables may be due to the limited variability in research study design as this variable has mostly been assessed in studies using a similar massage treatment regimen with assessment at comparable time points. No assessment of the catecholamines has been done in plasma samples with massage therapy.

Comparison of our findings with a meta-analysis conducted by Moyer et al. (48) in 2004 yielded a similar conclusion for reduction in heart rate following a single massage treatment. However, in their conclusion they did not detect a single massage session change in salivary cortisol, but did find a change for blood pressure, which contrast with our findings. In our review, we excluded studies where massage therapy was administered from a therapist without formal training as well as studies that were not published in a peer-reviewed journal. In addition, our search process yielded several studies that were not included in the Moyer et al. analysis. This variance in article inclusion likely accounts for much of the inferential differences between the reviews.

The findings that emerged from the present review, however, may say less about the true clinical effects of massage therapy on stress measures than they do about the current state of massage therapy research. In general, the studies we reviewed revealed a variety of methodological shortcomings. Although many of the studies were RCTs, several of those reports failed to include data from a control group for one or more of the selected outcome measures. Other studies using a RCT design showed statistically significant differences in outcome measures of interest between the intervention and control groups at baseline, but did not address the possible reasons for these differences (e.g. failure of the randomization process) or control for this difference in the analysis. Several studies presented results in a manner that made it difficult for the reviewers to even interpret the statistical findings. In addition, control groups in some studies were not always given an equivalent time for the control assignment such as reading, relaxation breathing or other quiet time activity. While RCTs are critical to determine the efficacy of massage therapy and other therapeutic interventions, it is equally critical that these trials be conducted with methodological rigor to ensure the validity and reliability of their results, and that these results be presented in a manner that can be clearly understood by the research community and massage 
therapy practitioners. Several of the studies we reviewed failed in this regard.

The studies meeting our inclusion criteria encompassed a wide variety of massage therapy interventions and an even more diverse group of subjects. The length, duration and treatment protocol were seldom justified by the study authors with regard to the specific needs of the treatment population and interventions ranged widely in both session and study duration. Subject populations ranged from healthy adults to pain patients to HIV-positive patients and victims of sexual abuse. Rarely did we find more than one study on one particular population; a healthy population was the most frequently studied. Furthermore, for those experiencing stress, most participants had been experiencing chronic stress associated with a condition they likely had for many years; populations experiencing acute stress were less frequently reported. All of these studies had relatively few participants with a range from 5 to 105 subjects $($ median $=30$ ). There is no doubt that small clinical trials are important for providing proof of concept and justifying the pursuit of larger studies that can more definitively assess the effects of massage intervention on stress in a particular patient population. Unfortunately, to date, the research on massage therapy and stress has not progressed to demonstrate efficacy in a trial of sufficient size or methodological rigor to make definitive statements about its efficacy in reducing stress as measured by physiological variables in any particular patient population.

Future research efforts to examine the effectiveness of massage therapy in alleviating physiological symptoms of stress should employ methodological rigor and integrity so as to increase public and scientific confidence in the published results. Reports of statistical findings must be comprehensive and clearly presented. Massage therapy protocols should also be clearly documented for the purposes of scientific replication and differentiating the effectiveness of various interventions. Smaller studies should be conducted with an eye toward large RCTs that provide more definitive results on the effectiveness of massage therapy in alleviating stress. Ideally, these trials would test outcome measures that have clinical, not just statistical, significance; answer questions regarding efficacy of different massage therapy modalities; employ different modes of delivery (e.g. chair versus table); account for intertherapist variations and interactions between different massage therapy modalities; and thoroughly examine effects on different clinical and nonclinical populations. The findings that would emerge from these more meticulous and comprehensive research approaches would identify models that have the potential to yield more interpretable results, thus increasing confidence and validating the perceived benefits of massage therapy for stress management.
Conflict of interest: None declared.

\section{Acknowledgements}

The Massage Therapy Foundation provided financial support for this review article. The authors are especially grateful to G Hymel, A Pittas, J Balletto, A Low Choy and $\mathbf{J}$ Ryan for their assistance on this project.

\section{References}

1. Peddicord K. Strategies for promoting stress reduction and relaxation. Nurs Clin North Am 1991;26:867-74.

2. Clancy CP, Graybeal A, Tompson WP, Badgett KS, Feldman ME, Calhoun PS, et al. Lifetime trauma exposure in veterans with military-related posttraumatic stress disorder: association with current symptomatology. J Clin Psychiatry 2006;67:1346-53.

3. Scocco P, Barbieri I, Frank E. Interpersonal problem areas and onset of panic disorder. Psychopathology 2007;40:8-13.

4. Samuels-Dennis J. Relationship among employment status, stressful life events, and depression in single mothers. Can J Nurs Res 2006;38:58-80

5. Bech P, Andersen MB, Bech-Andersen G, Tonnesen S, Agnarsdottir E, Borg V. Work-related stressors, depression and quality of life in Danish managers. Eur Psychiatry 2005;20 (Suppl 3): S318-25.

6. Marsland AL, Bachen EA, Cohen S, Rabin B, Manuck SB. Stress, immune reactivity and susceptibility to infectious disease. Physiol Behav 2002;77:711-6.

7. Sapolsky RM. Why Zebras don't get Ulcers, 3rd edn. New York: Times Books, 2004.

8. Weiss BL. Eliminating Stress, Finding Inner Peace. Carlsbad, CA: Hay House, 2003.

9. Eisenberg DM, Davis RB, Ettner SL, Appel S, Wilkey S, Van Rompay M, et al. Trends in alternative medicine use in the United States, 1990-1997: results of a follow-up national survey. JAMA 1998;280:1569-75.

10. Tindle HA, Davis RB, Phillips RS, Eisenberg DM. Trends in use of complementary and alternative medicine by US adults: 1997-2002. Altern Ther Health Med 2005;11:42-9.

11. Barnes PM, Powell-Griner E, McFann K, Nahin RL. Complementary and alternative medicine use among adults: United States, 2002. Adv Data 2004:1-19.

12. Cherkin DC, Deyo RA, Sherman KJ, Hart LG, Street JH, Hrbek A, et al. Characteristics of visits to licensed acupuncturists, chiropractors, massage therapists, and naturopathic physicians. $J$ Am Board Fam Pract 2002;15:463-72.

13. de Rooij SR, Painter RC, Phillips DI, Osmond C, Tanck MW, Bossuyt PM, et al. Cortisol responses to psychological stress in adults after prenatal exposure to the Dutch famine. Psychoneuroendocrinology 2006;31:1257-65.

14. Armario A. The hypothalamic-pituitary-adrenal axis: what can it tell us about stressors? CNS Neurol Disord Drug Targets 2006;5:485-501.

15. Sherman KJ, Cherkin DC, Kahn J, Erro J, Hrbek A, Deyo RA, et al. A survey of training and practice patterns of massage therapists in two US states. BMC Complement Altern Med 2005;5:13.

16. Long L, Huntley A, Ernst E. Which complementary and alternative therapies benefit which conditions? A survey of the opinions of 223 professional organizations. Complement Ther Med 2001;9:178-85.

17. Keegan L. Therapies to reduce stress and anxiety. Crit Care Nurs Clin North Am 2003;15:321-7.

18. Moraska A. Therapist education impacts the massage effect on postrace muscle recovery. Med Sci Sports Exerc 2007;39:34-7.

19. Jadad AR, Moore RA, Carroll D, Jenkinson C, Reynolds DJ, Gavaghan DJ, et al. Assessing the quality of reports of randomized clinical trials: is blinding necessary? Control Clin Trials 1996;17:1-12.

20. Moher D, Jadad AR, Tugwell P. Assessing the quality of randomized controlled trials. Current issues and future directions. Int J Technol Assess Health Care 1996;12:195-208. 
21. Field T, Hernandez Reif M, Hart S, Quintino O, Drose LA, Field $\mathrm{T}$, et al. Effects of sexual abuse are lessened by massage therapy. J Bodywork Movement Ther 1997;1:65-9.

22. Field T, Schanberg S, Kuhn C, Fierro K, Henteleff T, Mueller C, et al. Bulimic adolescents benefit from massage therapy. Adolescence 1998;33:555-63.

23. Hart S, Field T, Hernandez Reif M, Nearing G, Shaw S, Schanberg S, et al. Anorexia nervosa symptoms are reduced by massage therapy. Eating Disord 2001;9:289-99.

24. Field T, Peck M, Krugman S, Tuchel T, Schanberg S, Kuhn C, et al. Burn injuries benefit from massage therapy. J Burn Care Rehabil 1998;19:241-4.

25. Lawler SP, Cameron LD. A randomized, controlled trial of massage therapy as a treatment for migraine. Ann Behav Med 2006;32:50-9.

26. Hernandez-Reif M, Field T, Krasnegor J, Theakston H. Lower back pain is reduced and range of motion increased after massage therapy. Int J Neurosci 2001;106:131-45.

27. Hernandez-Reif M, Field T, Krasnegor J, Theakston H, Hossain Z, Burman I. High blood pressure and associated symptoms were reduced by massage therapy. $J$ Bodywork Movement Ther 2000;4:31-38.

28. Olney CM. The effect of therapeutic back massage in hypertensive persons: a preliminary study. Biol Res Nurs 2005;7:98-105.

29. Ironson G, Field T, Scafidi F, Hashimoto M, Kumar M, Kumar A, et al. Massage therapy is associated with enhancement of the immune system's cytotoxic capacity. Int J Neurosci 1996;84:205-17.

30. Ahles TA, Tope DM, Pinkson B, Walch S, Hann D, Whedon M, et al. Massage therapy for patients undergoing autologous bone marrow transplantation. J Pain Symptom Manage 1999;18:157-63.

31. Hernandez Reif $M$, Ironson $G$, Field $T$, Hurley $J$, Katz G, Diego M, et al. Breast cancer patients have improved immune and neuroendocrine functions following massage therapy. $J$ Psychosom Res 2004;57:45-52.

32. Goodfellow LM. The effects of therapeutic back massage on psychophysiologic variables and immune function in spouses of patients with cancer. Nurs Res 2003;52:318-328.

33. Taylor AG, Galper DI, Taylor P, Rice LW, Andersen W, Irvin W, et al. Effects of adjunctive Swedish massage and vibration therapy on short-term postoperative outcomes: a randomized, controlled trial. J Altern Complement Med 2003;9:77-89.

34. Hayes J, Cox C. Immediate effects of a five-minute foot massage on patients in critical care. Complement Ther Nurs Midwifery 2000;6:9-13

35. Aourell M, Skoog M, Carleson J. Effects of Swedish massage on blood pressure. Complement Ther Clin Pract 2005;11:242-6.

36. Bost N, Wallis $\mathrm{M}$. The effectiveness of a 15 minute weekly massage in reducing physical and psychological stress in nurses. Aust $J$ Adv Nurs 2006;23:28-33.
37. Cady SH, Jones GE. Massage therapy as a workplace intervention for reduction of stress. Percept Mot Skills 1997;84:157-8.

38. Cowen VS, Burkett L, Bredimus J, Evans DR, Lamey S, Neuhauser $\mathrm{T}$, et al. A comparative study of Thai massage and Swedish massage relative to physiological and psychological measures. J Bodywork Movement Ther 2006;10:266-75.

39. Diego MA, Field T, Sanders C, Hernandez-Reif M. Massage therapy of moderate and light pressure and vibrator effects on EEG and heart rate. Int $J$ Neurosci 2004;114:31-44.

40. Field T, Ironson G, Scafidi F, Nawrocki T, Goncalves A, Burman I, et al. Massage therapy reduces anxiety and enhances EEG pattern of alertness and math computations. Int $J$ Neurosci 1996;86:197-205.

41. Leivadi S, Hernandez Reif M, Field T, O'Rourke M, D'Arienzo S, Lewis $\mathrm{D}$, et al. Massage therapy and relaxation effects on university dance students. J Dance Med Sci 1999;3:108-12.

42. Delaney J, Leong KS, Watkins A, Brodie D. The short-term effects of myofascial trigger point massage therapy on cardiac autonomic tone in healthy subjects. $J$ Adv Nurs 2002;37:364-71.

43. Hernandez Reif M, Field T, Largie S, Cullen C, Beutler J, Sanders C, et al. Parkinson's disease symptoms are differentially affected by massage therapy vs. progressive muscle relaxation: a pilot study. J Bodywork Movement Ther 2002;6:177-182.

44. Andersson K, Wandell P, Tornkvist L. Tactile massage improves glycaemic control in women with type 2 diabetes: a pilot study. Pract Diab Int 2004;21:105-09.

45. McKechnie A, Wilson F, Watson N, Scott D. Anxiety states: a preliminary report on the value of connective tissue massage. $J$ Psychosom Res 1983;27:125-29.

46. Gozansky WS, Lynn JS, Laudenslager ML, Kohrt WM. Salivary cortisol determined by enzyme immunoassay is preferable to serum total cortisol for assessment of dynamic hypothalamic-pituitaryadrenal axis activity. Clin Endocrinol (Oxf) 2005;63:336-41.

47. Lundberg U. Catecholamines and environmental stress. 2003. http://www.macses.ucsf.edu/Research/Allostatic/notebook/catecholamine. html (accessed December 5, 2007).

48. Moyer CA, Rounds J, Hannum JW. A meta-analysis of massage therapy research. Psychol Bull 2004;130:3-18.

49. Wyrwich KW, Wolinsky FD. Identifying meaningful intra-individual change standards for health-related quality of life measures. $J$ Eval Clin Pract 2000;6:39-49.

50. Coeytaux RR, Kaufman JS, Chao R, Mann JD, Devellis RF. Four methods of estimating the minimal important difference score were compared to establish a clinically significant change in Headache Impact Test. J Clin Epidemiol 2006;59:374-80.

Received September 7, 2007; accepted April 4, 2008 


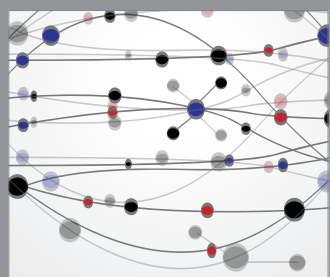

The Scientific World Journal
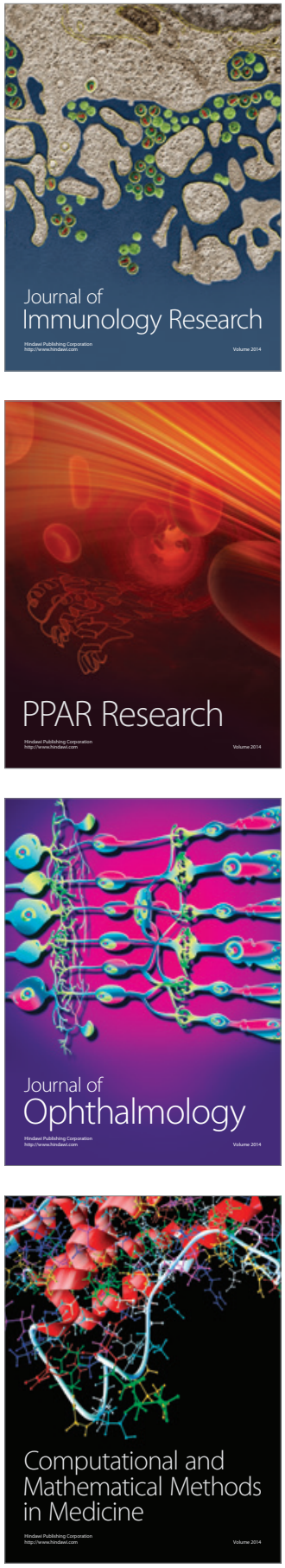

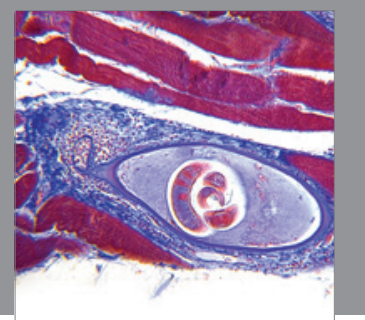

Gastroenterology

Research and Practice
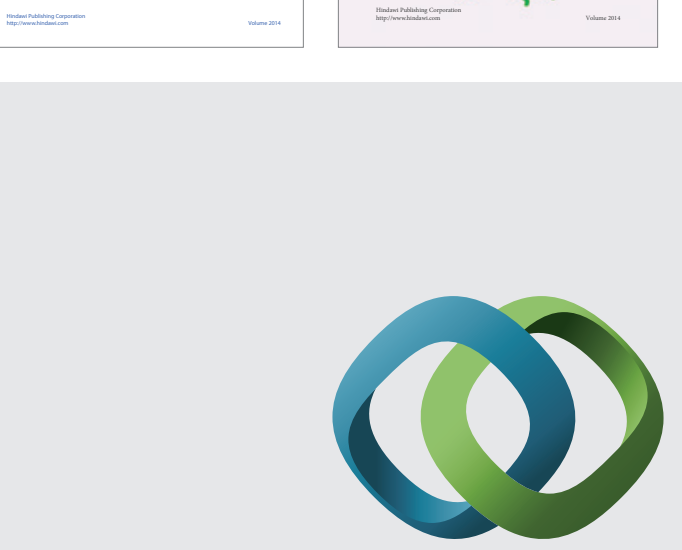

\section{Hindawi}

Submit your manuscripts at

http://www.hindawi.com
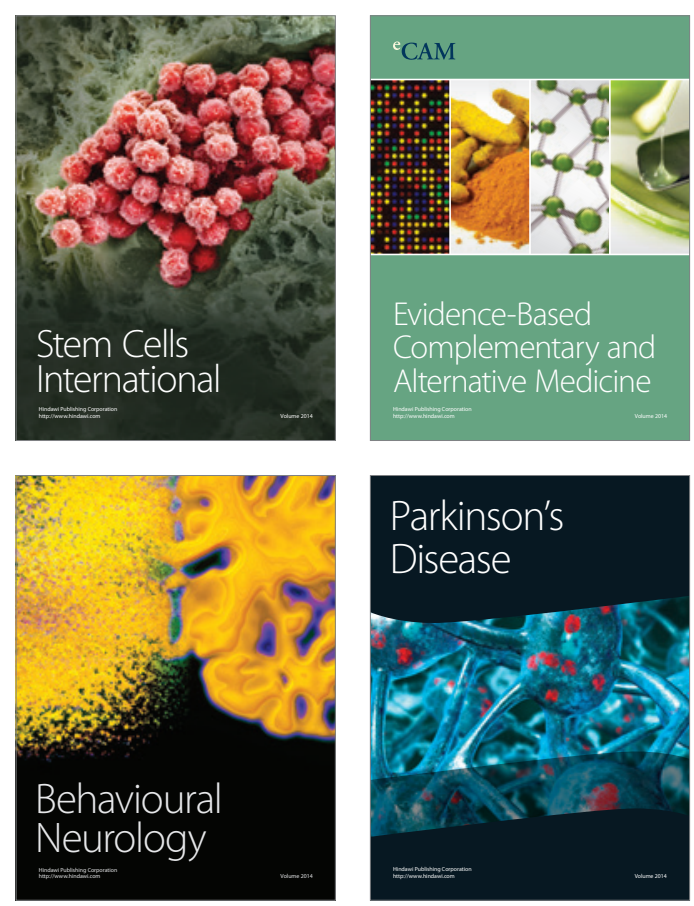

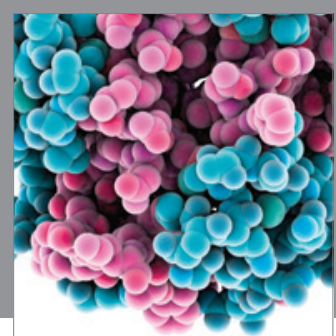

Journal of
Diabetes Research

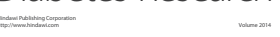

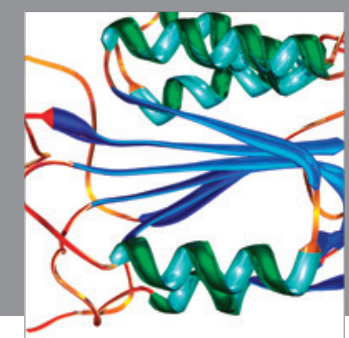

Disease Markers
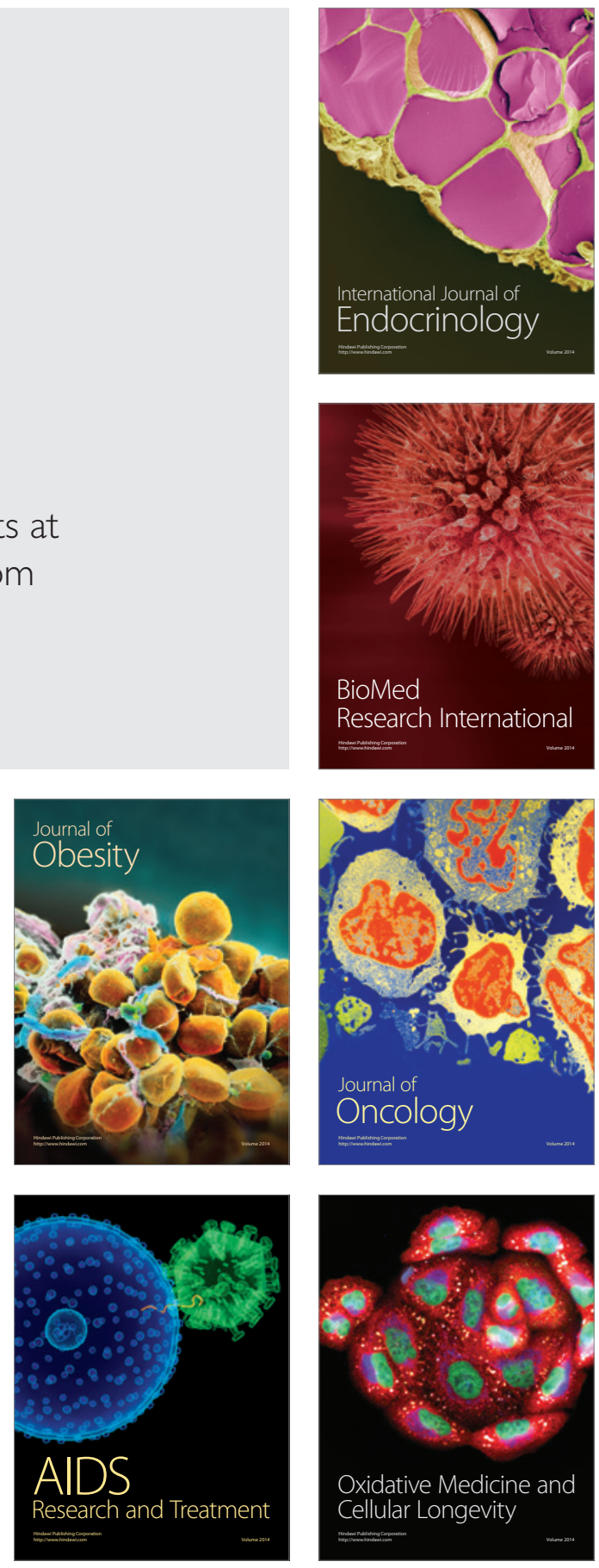